\title{
Existence Results and the Monotone Iterative Technique for Nonlinear Fractional Differential Systems with Coupled Four-Point Boundary Value Problems
}

\author{
Yujun Cui ${ }^{1}$ and Yumei Zou' ${ }^{2}$ \\ ${ }^{1}$ Department of Mathematics, Shandong University of Science and Technology, Qingdao 266590, China \\ ${ }^{2}$ Department of Statistics and Finance, Shandong University of Science and Technology, Qingdao 266590, China \\ Correspondence should be addressed to Yujun Cui; cyj720201@163.com
}

Received 13 May 2014; Accepted 11 July 2014; Published 23 July 2014

Academic Editor: Shurong Sun

Copyright (c) 2014 Y. Cui and Y. Zou. This is an open access article distributed under the Creative Commons Attribution License, which permits unrestricted use, distribution, and reproduction in any medium, provided the original work is properly cited.

By establishing a comparison result and using the monotone iterative technique combined with the method of upper and lower solutions, we investigate the existence of solutions for nonlinear fractional differential systems with coupled four-point boundary value problems.

\section{Introduction}

This paper discusses the coupled four-point boundary value problems

$$
\begin{gathered}
D^{p} x(t)+f(t, x(t), y(t))=0, \quad t \in(0,1), 1<p \leq 2, \\
D^{q} y(t)+g(t, x(t), y(t))=0, \quad t \in(0,1), 1<q \leq 2, \\
x(0)=y(0)=0, \quad x(1)=a y(\xi), \quad y(1)=b x(\eta),
\end{gathered}
$$

where $f$ and $g:(0,1) \times \mathbb{R} \times \mathbb{R} \rightarrow \mathbb{R}$ are continuous, $\xi, \eta \in$ $(0,1), a, b>0$ with $a b<1$, and $D^{p} x$ denotes the Caputo fractional derivative of $x$ with $1<p \leq 2$ defined by

$$
D^{p} x(t)=I^{2-p} x^{\prime \prime}(t)=\frac{1}{\Gamma(p)} \int_{0}^{t}(t-s)^{1-p} x^{\prime \prime}(s) d s
$$

$I^{2-p}$ is the Riemann-Liouville fractional integral of order $2-p$; see $[1-4]$.
It is well known that

$$
\begin{gathered}
I^{2-p}\left(D^{2-p} x(t)\right)=x(t)-\sum_{k=0}^{1} \frac{x^{(k)}\left(0^{+}\right)}{k !} t^{k}, \\
D^{2-p}\left(I^{2-p} x(t)\right)=x(t) .
\end{gathered}
$$

Fractional differential equation's modeling capabilities in physics, chemistry, economics, and other fields, over the last few decades, have resulted in the rapid development of the theory of fractional differential equations; we refer the reader to the books [1-4]. On the other hand, the study of systems involving coupled boundary value problems is also important as such systems occur in the study of reaction-diffusion equations and Sturm-Liouville problems, for example, [5-16]. In [17-25], using the upper and lower solutions method and the monotone iterative method, the authors considered the existence of solutions of initial value problems and boundary value problems for fractional differential equations. But, as far as we know, there have been few papers which have considered the existence of solutions of (1) by means of the monotone iterative method.

Motivated by the above papers, in this paper, we will investigate the existence of a solution of problem (1) by means of the upper and lower solutions method and the monotone 
iterative method. The novelty of this paper is that Caputotype fractional differential systems involve two different fractional derivatives $D^{p}$ and $D^{q}$ and that the nonlinear terms $f, g$ in the systems (1) involve unknown functions $x(t)$ and $y(t)$.

In the following, we denote

$$
E=C^{2}([0,1], \mathbb{R}), \quad E_{1}=C([0,1],(0,+\infty)) .
$$

\section{Preliminaries and Lemmas}

In this section, we introduce the definition of the lower and upper solutions and present some existence and uniqueness results for linear problems together with comparison results for differential systems (1) which will be needed in the next section.

Throughout this paper, we always assume that the following condition is satisfied:

$\left(H_{1}\right) 0<a b<1$.

Definition 1. $\left(u_{0}, v_{0}\right) \in E \times E$ is called a lower system of solutions of differential system (1) if

$$
\begin{gathered}
D^{p} u_{0}(t)+f\left(t, u_{0}(t), v_{0}(t)\right) \geq 0, \quad t \in(0,1), \\
D^{q} v_{0}(t)+g\left(t, u_{0}(t), v_{0}(t)\right) \geq 0, \quad t \in(0,1), \\
u_{0}(0) \leq 0, \quad v_{0}(0) \leq 0, \\
u_{0}(1) \leq a v_{0}(\xi), \quad v_{0}(1) \leq b u_{0}(\eta) .
\end{gathered}
$$

Analogously, $\left(\alpha_{0}, \beta_{0}\right) \in E \times E$ is called an upper system of solutions if it satisfies the reversed inequalities.

If $u_{0}(t) \leq \alpha_{0}(t)$ and $v_{0}(t) \leq \beta_{0}(t), t \in[0,1]$, we say that $\left(u_{0}, v_{0}\right)$ and $\left(\alpha_{0}, \beta_{0}\right)$ are ordered lower and upper system of solutions of (1). In what follows, we assume that $\left(u_{0}, v_{0}\right)$ and $\left(\alpha_{0}, \beta_{0}\right)$ are ordered lower and upper system of solutions of (1) and define the sector

$$
\begin{aligned}
\Omega=\{(x, y) \in E \times E & :\left(u_{0}(t), v_{0}(t)\right) \\
\leq & (x(t), y(t)) \\
\leq & \left.\left(\alpha_{0}(t), \beta_{0}(t)\right), t \in[0,1]\right\},
\end{aligned}
$$

where the vectorial inequalities mean that the same inequalities hold between their corresponding components.

Lemma 2 (see [17]). Let $z(t) \in E$ and $r(t) \in E_{1}$. If $z(t)$ satisfies the inequality

$$
\begin{gathered}
-D^{p} z(t) \leq-r(t) z(t), \quad p \in(1,2], t \in(0,1), \\
z(0) \leq 0, \quad z(1) \leq 0,
\end{gathered}
$$

then $z(t) \leq 0, \forall t \in[0,1]$.
We have the following important result.

Lemma 3 (comparison theorem). Let $M(t), N(t) \in E_{1}$ be given. Assume that $x(t), y(t)$ satisfy

$$
\begin{aligned}
& -D^{p} x(t) \leq-M(t) x(t), \quad t \in(0,1), \\
& -D^{q} y(t) \leq-N(t) y(t), \quad t \in(0,1), \\
& x(0) \leq 0, \quad y(0) \leq 0, \\
& x(1) \leq a y(\xi), \quad y(1) \leq b x(\eta) .
\end{aligned}
$$

Then $x(t) \leq 0, y(t) \leq 0, \forall t \in[0,1]$.

Proof. Suppose the contrary. By Lemma 2, We consider the following three possible cases.

Case 1. Consider $x(1) \leq 0$ and $y(1)>0$. By Lemma $2, x(t) \leq$ $0, \forall t \in[0,1]$. Then $y(1) \leq b x(\eta) \leq 0$ which contradicts $y(1)>0$.

Case 2. Consider $y(1) \leq 0$ and $x(1)>0$. By Lemma 2, $y(t) \leq$ $0, \forall t \in[0,1]$. Then $x(1) \leq a y(\xi) \leq 0$ which contradicts $x(1)>$ 0 .

Case 3. Consider $x(1)>0$ and $y(1)>0$. By Lemma 2, we have $x(1)=\max _{t \in[0,1]} x(t)>0$ and $y(1)=\max _{t \in[0,1]} y(t)>0$. We only prove that $x(1)=\max _{t \in[0,1]} x(t)>0$. If not, $x(t)$ has a local positive maximum at some $t_{0} \in(0,1)$ such that $x\left(t_{0}\right)=\max _{t \in[0,1]} x(t)>0$. Then, by Theorem 2.1 in [21], we have the fact that the Caputo derivative of the function $x$ is nonpositive at the point $t_{0}$. Thus,

$$
0 \leq-D^{p} x\left(t_{0}\right) \leq-M\left(t_{0}\right) x\left(t_{0}\right)<0,
$$

which is a contradiction. Furthermore, considering the boundary condition $y(1) \leq b x(\eta)$, there exists $t_{1} \in[0, \eta)$ such that

$$
x(t) \leq 0, \quad t \in\left[0, t_{1}\right] ; \quad x(t) \geq 0, \quad t \in\left[t_{1}, 1\right] .
$$

A similar proof, for $y(t)$, gives us that there exists $t_{2} \in[0, \xi)$ such that

$$
y(t) \leq 0, \quad t \in\left[0, t_{2}\right] ; \quad y(t) \geq 0, \quad t \in\left[t_{2}, 1\right] .
$$

It follows from (10) and (11) that

$$
x(1) \leq a y(\xi) \leq a y(1) \leq a b x(\eta) \leq a b x(1),
$$

which implies that $a b \geq 1$, a contradiction. Hence $x(t) \leq$ $0, y(t) \leq 0, \forall t \in[0,1]$.

Corollary 4. Let $M(t), N(t) \in E_{1}$ be given. Assume that $x(t), y(t)$ satisfy

$$
\begin{gathered}
-D^{p} x(t)=-M(t) x(t), \quad t \in(0,1), \\
-D^{q} y(t)=-N(t) y(t), \quad t \in(0,1), \\
x(0)=0, \quad y(0)=0, \\
x(1)=a y(\xi), \quad y(1)=b x(\eta) .
\end{gathered}
$$

Then $x(t)=y(t)=0, \forall t \in[0,1]$. 
Lemma 5. Let $\rho, \sigma \in C[0,1]$, then the linear differential system with coupled four-point boundary value problem

$$
\begin{gathered}
D^{p} x(t)+\rho(t)=0, \quad t \in(0,1), 1<p \leq 2, \\
D^{q} y(t)+\sigma(t)=0, \quad t \in(0,1), 1<q \leq 2, \\
x(0)=y(0)=0, \quad x(1)=a y(\xi), \quad y(1)=b x(\eta)
\end{gathered}
$$

has integral representation

$$
\begin{aligned}
& x(t)=\int_{0}^{1} G_{1}(t, s) \rho(s) d s+\int_{0}^{1} H_{1}(t, s) \sigma(s) d s, \\
& y(t)=\int_{0}^{1} G_{2}(t, s) \sigma(s) d s+\int_{0}^{1} H_{2}(t, s) \rho(s) d s,
\end{aligned}
$$

where

$$
\begin{gathered}
G_{1}(t, s)=G_{p}(t, s)+\frac{a b \xi t}{1-a b \xi \eta} G_{p}(\eta, s), \\
H_{1}(t, s)=\frac{a t}{1-a b \xi \eta} G_{q}(\xi, s), \\
G_{2}(t, s)=G_{q}(t, s)+\frac{a b \eta t}{1-a b \xi \eta} G_{q}(\xi, s), \\
H_{2}(t, s)=\frac{b t}{1-a b \xi \eta} G_{p}(\eta, s), \\
G_{p}(t, s)= \begin{cases}\frac{t(1-s)^{p-1}-(t-s)^{p-1}}{\Gamma(p)}, & 0 \leq s \leq t \leq 1, \\
\frac{t(1-s)^{p-1}}{\Gamma(p)}, & 0 \leq t \leq s \leq 1 .\end{cases}
\end{gathered}
$$

Proof. It follows from [21] that (14) is equivalent to the system of integral equations

$$
\begin{array}{ll}
x(t)=x(1) t+\int_{0}^{1} G_{p}(t, s) \rho(s) d s, & t \in[0,1], \\
y(t)=y(1) t+\int_{0}^{1} G_{q}(t, s) \sigma(s) d s, & t \in[0,1] .
\end{array}
$$

By coupled four-point boundary value conditions of problem (14), we have

$$
\begin{aligned}
& y(1)=b x(\eta)=b \eta x(1)+b \int_{0}^{1} G_{p}(\eta, s) \rho(s) d s, \\
& x(1)=a y(\xi)=a \xi y(1)+a \int_{0}^{1} G_{q}(\xi, s) \sigma(s) d s .
\end{aligned}
$$

After simple computation, we get

$$
\begin{aligned}
x(1)= & \frac{a b \xi}{1-a b \xi \eta} \int_{0}^{1} G_{p}(\eta, s) \rho(s) d s \\
& +\frac{a}{1-a b \xi \eta} \int_{0}^{1} G_{q}(\xi, s) \sigma(s) d s, \\
y(1)= & \frac{a b \eta}{1-a b \xi \eta} \int_{0}^{1} G_{q}(\xi, s) \sigma(s) d s \\
& +\frac{b}{1-a b \xi \eta} \int_{0}^{1} G_{p}(\eta, s) \rho(s) d s .
\end{aligned}
$$

Substituting (20) into (18) and (21) into (19), respectively, we obtain the desired results.

Now we enunciate the following existence and uniqueness results for differential system:

$$
\begin{aligned}
& D^{p} x(t)-M(t) x(t)+\rho(t)=0, \quad t \in(0,1), 1<p \leq 2, \\
& D^{q} y(t)-N(t) y(t)+\sigma(t)=0, \quad t \in(0,1), 1<q \leq 2, \\
& x(0)=y(0)=0, \quad x(1)=a y(\xi), \quad y(1)=b x(\eta),
\end{aligned}
$$

where $M, N \in E_{1}$.

Lemma 6. Let $M, N \in E_{1}$. Then differential system (22) has a unique solution.

Proof. Indeed, by Lemma 5, differential system (22) is equivalent to the operator equation

$$
(x, y)=T(x, y)+(\tilde{\rho}, \tilde{\sigma}),
$$

where

$$
\begin{aligned}
& T(x, y)(t) \\
& =\left(-\int_{0}^{1} G_{1}(t, s) M(s) x(s) d s-\int_{0}^{1} H_{1}(t, s) N(s) y(s) d s\right. \\
& \quad-\int_{0}^{1} G_{2}(t, s) N(s) y(s)(s) d s \\
& \left.\quad-\int_{0}^{1} H_{2}(t, s) M(s) x(s) d s\right) \\
& \tilde{\rho}(t)=\int_{0}^{1} G_{1}(t, s) \rho(s) d s+\int_{0}^{1} H_{1}(t, s) \sigma(s) d s \\
& \tilde{\sigma}(t)=\int_{0}^{1} G_{2}(t, s) \sigma(s) d s+\int_{0}^{1} H_{2}(t, s) \rho(s) d s .
\end{aligned}
$$

We apply the Fredholm theorem to find the unique solution of differential system (22). By using standard arguments, we can easily show that $T: C[0,1] \times C[0,1] \rightarrow C[0,1] \times$ $C[0,1]$ is linear completely continuous Also, by Corollary 4 , the operator equation $(x, y)=T(x, y)$ has only the zero solution. Thus, for given $(\widetilde{\rho}, \widetilde{\sigma}) \in C[0,1] \times C[0,1]$, operator equation (23) has a unique solution in $C[0,1] \times C[0,1]$, by the Fredholm theorem. This ends the proof. 


\section{Main Results}

In this section, we prove the existence of extremal solutions of differential system (1).

Theorem 7. Assume that $f \in C([0,1] \times \mathbb{R} \times \mathbb{R}, \mathbb{R}), g \in$ $C([0,1] \times \mathbb{R} \times \mathbb{R}, \mathbb{R})$. Let $\left(u_{0}, v_{0}\right)$ and $\left(\alpha_{0}, \beta_{0}\right)$ be ordered lower and upper system of solutions of (1). In addition, we assume that

$\left(H_{2}\right) f(t, x, y)$ is nondecreasing in $y$ and there exists $M(t) \in E_{1}$ such that

$$
f\left(t, x_{1}, y\right)-f\left(t, x_{2}, y\right) \geq-M(t)\left(x_{1}-x_{2}\right),
$$

where $u_{0}(t) \leq x_{2} \leq x_{1} \leq \alpha_{0}(t), v_{0}(t) \leq y \leq \beta_{0}(t)$

$\left(H_{3}\right) g(t, x, y)$ is nondecreasing in $x$ and there exists $N(t) \epsilon$ $E_{1}$ such that

$$
g\left(t, x, y_{1}\right)-g\left(t, x, y_{2}\right) \geq-N(t)\left(y_{1}-y_{2}\right),
$$

where $v_{0}(t) \leq y_{2} \leq y_{1} \leq \beta_{0}(t), u_{0}(t) \leq x \leq \alpha_{0}(t)$.

Then differential system (1) has extremal solutions in the section $\Omega$.

Proof. Let us define two sequences $\left\{\left(u_{n}, v_{n}\right),\left(\alpha_{n}, \beta_{n}\right)\right\}$ by relations

$$
\begin{gathered}
D^{p} u_{n+1}(t)-M(t) u_{n+1}(t)+f\left(t, u_{n}(t), v_{n}(t)\right) \\
+M(t) u_{n}(t)=0, \quad t \in(0,1) \\
D^{q} v_{n+1}(t)-N(t) v_{n+1}(t)+g\left(t, u_{n}(t), v_{n}(t)\right) \\
+N(t) v_{n}(t)=0, \quad t \in(0,1), \\
u_{n+1}(0)=v_{n+1}(0)=0, \quad u_{n+1}(1)=a v_{n+1}(\xi), \\
\quad v_{n+1}(1)=b u_{n+1}(\eta) \\
D^{p} \alpha_{n+1}(t)-M(t) \alpha_{n+1}(t)+f\left(t, \alpha_{n}(t), \beta_{n}(t)\right) \\
+M(t) \alpha_{n}(t)=0, \quad t \in(0,1), \\
D^{q} \beta_{n+1}(t)-N(t) \beta_{n+1}(t)+g\left(t, \alpha_{n}(t), \beta_{n}(t)\right) \\
+N(t) \beta_{n}(t)=0, \quad t \in(0,1), \\
\alpha_{n+1}(0)=\beta_{n+1}(0)=0, \quad \alpha_{n+1}(1)=a \beta_{n+1}(\xi), \\
\beta_{n+1}(1)=b \alpha_{n+1}(\eta)
\end{gathered}
$$

for $n=1,2, \ldots$. Note that $\left\{\left(u_{1}, v_{1}\right),\left(\alpha_{1}, \beta_{1}\right)\right\}$ are well defined, by Lemma 6 . First, we show that

$$
\left(u_{0}, v_{0}\right) \leq\left(u_{1}, v_{1}\right) \leq\left(\alpha_{1}, \beta_{1}\right) \leq\left(\alpha_{0}, \beta_{0}\right) .
$$

Let $z=u_{0}-u_{1}, w=v_{0}-v_{1}$. This and the assumption that $\left(u_{0}, v_{0}\right)$ is a lower system of solutions of (1) yield

$$
\begin{gathered}
-D^{p} z(t) \leq-M(t) z(t), \quad t \in(0,1), \\
-D^{q} w(t) \leq-N(t) w(t), \quad t \in(0,1), \\
z(0) \leq 0, \quad w(0) \leq 0, \quad z(1) \leq a w(\xi), \\
w(1) \leq b z(\eta) .
\end{gathered}
$$

Hence, $u_{0}(t) \leq u_{1}(t)$ and $v_{0}(t) \leq v_{1}(t), t \in[0,1]$, by Lemma 3 . By a similar way, we can show that $\alpha_{1}(t) \leq \alpha_{0}(t)$ and $\beta_{1}(t) \leq$ $\beta_{0}(t), t \in[0,1]$. Now we put $z=u_{1}-\alpha_{1}, w=v_{1}-\beta_{1}$. Hence, in view of assumptions $\left(\mathrm{H}_{2}\right),\left(\mathrm{H}_{3}\right)$, we have

$$
\begin{aligned}
&-D^{p} z(t)=-M(t) z(t)-f\left(t, \alpha_{0}(t), \beta_{0}(t)\right) \\
&-M(t) \alpha_{0}(t)+f\left(t, u_{0}(t), v_{0}(t)\right)+M(t) u_{0}(t) \\
& \leq-M(t) z(t)-f\left(t, \alpha_{0}(t), \beta_{0}(t)\right) \\
&-M(t) \alpha_{0}(t)+f\left(t, u_{0}(t), \beta_{0}(t)\right)+M(t) u_{0}(t) \\
& \leq-M(t) z(t), \quad t \in(0,1), \\
&-D^{q} w(t) \leq-N(t) w(t), \quad t \in(0,1), \\
& z(0)= 0, \quad w(0)=0, \quad z(1)=a w(\xi), \\
& w(1)=b z(\eta) .
\end{aligned}
$$

This and Lemma 3 prove that $\left(u_{1}, v_{1}\right) \leq\left(\alpha_{1}, \beta_{1}\right), t \in[0,1]$, so, relation (28) holds.

Now we show that $\left(u_{1}, v_{1}\right)$ is a lower system of solution of problem (1). Note that

$$
\begin{gathered}
D^{p} u_{1}(t)+f\left(t, u_{1}(t), v_{1}(t)\right) \\
\geq M(t) u_{1}(t)-f\left(t, u_{0}(t), v_{0}(t)\right)-M(t) u_{0}(t) \\
+f\left(t, u_{1}(t), v_{0}(t)\right) \geq 0, \quad t \in(0,1) \\
D^{q} v_{1}(t)+g\left(t, u_{1}(t), v_{1}(t)\right) \geq 0 \quad t \in(0,1) \\
u_{1}(0)=v_{1}(0)=0, \quad u_{1}(1)=a v_{1}(\xi) \\
v_{1}(1)=b u_{1}(\eta)
\end{gathered}
$$

by assumptions $\left(H_{2}\right),\left(H_{3}\right)$. It proves that $\left(u_{1}, v_{1}\right)$ is a lower system of solution of (1). Similarly, we can prove that $\left(\alpha_{1}, \beta_{1}\right)$ is an upper system of solution of problem (1).

By mathematical induction we can show that

$$
\begin{aligned}
\left(u_{0}, v_{0}\right) & \leq\left(u_{1}, v_{1}\right) \leq \cdots \leq\left(u_{n}, v_{n}\right) \leq\left(\alpha_{n}, \beta_{n}\right) \\
& \leq \cdots \leq\left(\alpha_{1}, \beta_{1}\right) \leq\left(\alpha_{0}, \beta_{0}\right)
\end{aligned}
$$

for $t \in[0,1]$ and $n=1,2, \ldots$ Employing standard arguments we see that the sequences $\left\{\left(u_{n}, v_{n}\right),\left(\alpha_{n}, \beta_{n}\right)\right\}$ converge to their limit functions $\left(u_{*}, v_{*}\right),\left(\alpha^{*}, \beta^{*}\right)$, respectively. Indeed, $\left(u_{*}, v_{*}\right)$ and $\left(\alpha^{*}, \beta^{*}\right)$ are solutions of problem (1) and $\left(u_{0}(t), v_{0}(t)\right) \leq\left(u_{*}, v_{*}\right) \leq\left(\alpha^{*}, \beta^{*}\right) \leq\left(\alpha_{0}, \beta_{0}\right)$ on $[0,1]$.

We need to show now that $\left(u_{*}, v_{*}\right)$ and $\left(\alpha^{*}, \beta^{*}\right)$ are extremal solutions of problem (1) in the segment $\Omega$. To prove it, we assume that $(x, y)$ is another solution of problem (1) and $\left(u_{n}, v_{n}\right) \leq(x(t), y(t)) \leq\left(\alpha_{n}(t), \beta_{n}(t)\right), t \in[0,1]$ for some 
positive integer $n$. Put $z=u_{n+1}-x, w=v_{n+1}-y$. Hence, in view of assumptions $\left(\mathrm{H}_{2}\right),\left(\mathrm{H}_{3}\right)$, we have

$$
\begin{aligned}
&-D^{p} z(t)=-M(t) z(t)-f(t, x(t), y(t)) \\
&-M(t) x(t)+f\left(t, u_{n}(t), v_{n}(t)\right)+M(t) u_{n}(t) \\
& \leq-M(t) z(t)-f(t, x(t), y(t))-M(t) x(t) \\
&+f\left(t, u_{n}(t), y(t)\right)+M(t) u_{n}(t) \\
& \leq-M(t) z(t), \quad t \in(0,1), \\
&- D^{q} w(t) \leq-N(t) w(t), \quad t \in(0,1), \\
& z(0)= 0, \quad w(0)=0, \quad z(1)=a w(\xi), \\
& w(1)=b z(\eta) .
\end{aligned}
$$

Hence, $\left(u_{n+1}(t), v_{n+1}(t)\right) \leq(x(t), y(t)), t \in[0,1]$, by Lemma 3. By a similar way, we can show that $(x(t), y(t)) \leq$ $\left(\alpha_{n+1}(t), \beta_{n+1}(t)\right), t \in[0,1]$. So by induction, $\left(u_{n}(t), v_{n}(t)\right) \leq$ $(x(t), y(t)) \leq\left(\alpha_{n}(t), \beta_{n}(t)\right), t \in[0,1]$ on $[0,1]$ for all $n$. Taking the limit as $n \rightarrow+\infty$, we conclude $\left(u_{*}(t), v_{*}(t)\right) \leq$ $(x(t), y(t)) \leq\left(\alpha^{*}(t), \beta^{*}(t)\right), t \in[0,1]$. That is, $\left(u_{*}(t), v_{*}(t)\right)$ and $\left(\alpha^{*}(t), \beta^{*}(t)\right)$ are extremal systems of solutions of (1) in $\Omega$.

\section{Example}

Consider the following problems:

$$
\begin{gathered}
D^{5 / 4} x(t)+\sin t-2 x(t)+\frac{1}{8} y^{3}(t) t^{3}=0, \quad t \in(0,1), \\
D^{7 / 4} y(t)-y^{3}(t)+x^{2}(t)+1=0, \quad t \in(0,1), \\
x(0)=y(0)=0, \quad x(1)=\frac{1}{4} y\left(\frac{1}{2}\right), \\
y(1)=2 x\left(\frac{3}{4}\right) .
\end{gathered}
$$

Obviously,

$$
\begin{gathered}
f(t, x, y)=\sin t-2 x+\frac{1}{8} y^{3} t^{3}, \\
g(t, x, y)=-y^{3}+x^{2}+1 .
\end{gathered}
$$

Take $\left(u_{0}(t), v_{0}(t)\right)=(0,0),\left(\alpha_{0}(t), \beta_{0}(t)\right)=(t, 2)$; then

$$
\begin{gathered}
D^{5 / 4} u_{0}(t)+\sin t-2 u_{0}(t)+\frac{1}{8} v_{0}^{3}(t) t^{3}=\sin t \geq 0, \\
t \in(0,1), \\
D^{7 / 4} v_{0}(t)-v_{0}^{3}(t)+u_{0}^{2}(t)+1=1 \geq 0, \quad t \in(0,1), \\
u_{0}(0)=v_{0}(0)=0, \quad u_{0}(1)=\frac{1}{4} v_{0}\left(\frac{1}{2}\right),
\end{gathered}
$$

$$
\begin{gathered}
v_{0}(1)=2 u_{0}\left(\frac{3}{4}\right), \\
D^{5 / 4} \alpha_{0}(t)+\sin t-2 \alpha_{0}(t)+\frac{1}{8} \beta_{0}^{3}(t) t^{3} \\
=\sin t-2 t+t^{3} \leq 0, \quad t \in(0,1), \\
D^{7 / 4} \beta_{0}(t)-\beta_{0}^{3}(t)+\alpha_{0}^{2}(t)+1=-7+t^{2} \leq 0, \\
\left.\alpha_{0}(0)=0, \quad \beta_{0}(0) \geq 0,1\right), \\
\alpha_{0}(1) \geq \frac{1}{4} \beta_{0}\left(\frac{1}{2}\right), \quad \beta_{0}(1) \geq 2 \alpha_{0}\left(\frac{3}{4}\right) .
\end{gathered}
$$

It shows that $\left(u_{0}(t), v_{0}(t)\right)$ and $\left(\alpha_{0}(t), \beta_{0}(t)\right)$ are lower and upper systems of solutions of (34).

On the other hand, it is easy to verify that conditions $\left(\mathrm{H}_{2}\right)$, $\left(H_{3}\right)$ hold for $M(t)=2$ and $N(t)=12$.

By Theorem 7, problem (34) has an extremal system of solutions $\left(u_{*}(t), v_{*}(t)\right)$ and $\left(\alpha^{*}(t), \beta^{*}(t)\right)$, which can be obtained by taking limits from some iterative sequences.

\section{Conflict of Interests}

The authors declare that there is no conflict of interests regarding the publication of this paper.

\section{Acknowledgments}

The authors sincerely thank the reviewers for their valuable suggestions and useful comments that have led to the present improved version of the original paper. The Project is supported by the National Natural Science Foundation of China (11371221, 61304074), the Specialized Research Foundation for the Doctoral Program of Higher Education of China (20123705110001), the Program for Scientific Research Innovation Team in Colleges and Universities of Shandong Province, the Postdoctoral Science Foundation of Shandong Province (201303074), and Foundation of SDUST.

\section{References}

[1] A. A. Kilbas, H. M. Srivastava, and J. J. Trujillo, Theory and Applications of Fractional Differential Equations, vol. 204, Elsevier, Amsterdam, The Netherlands, 2006.

[2] V. Lakshmikantham, S. Leela, and J. Vasundhara, Theory of Fractional Dynamic Systems, Cambridge Academic Publishers, Cambridge, Mass, USA, 2009.

[3] I. Podlubny, Fractional Differential Equations, vol. 198 of Mathematics in Science and Engineering, Academic Press, New York, NY, USA, 1999.

[4] S. G. Samko, A. A. Kilbas, and O. I. Marichev, Fractional Integrals and Derivatives, Theory and Applications, Gordon and Breach Science Publishers, Yverdon, Switzerland, 1993.

[5] H. Amann, "Parabolic evolution equations with nonlinear boundary conditions," in Nonlinear Functional Analysis and Its 
Applications, Proceedings of Symposia in Pure Mathematics, pp. 17-27, Providence, RI, USA, 1986.

[6] H. Amann, "Parabolic evolution equations and nonlinear boundary conditions," Journal of Differential Equations, vol. 72, no. 2, pp. 201-269, 1988.

[7] N. A. Asif and R. A. Khan, "Positive solutions to singular system with four-point coupled boundary conditions," Journal of Mathematical Analysis and Applications, vol. 386, no. 2, pp. 848-861, 2012.

[8] Y. Cui and J. Sun, "On existence of positive solutions of coupled integral boundary value problems for a nonlinear singular superlinear differential system," Electronic Journal of Qualitative Theory of Differential Equations, no. 41, 13 pages, 2012.

[9] Y. Cui, L. Liu, and X. Zhang, "Uniqueness and existence of positive solutions for singular differential systems with coupled integral boundary value problems," Abstract and Applied Analysis, vol. 2013, Article ID 340487, 9 pages, 2013.

[10] Y. Cui and Y. Zou, "Monotone iterative method for differential systems with coupled integral boundary value problems," Boundary Value Problems, vol. 2013, article 245, 2013.

[11] K. Deng, "Blow-up rates for parabolic systems," Zeitschrift für Angewandte Mathematik und Physik, vol. 47, pp. 132-143, 1996.

[12] K. Deng, "Global existence and blow-up for a system of heat equations with non-linear boundary conditions," Mathematical Methods in the Applied Sciences, vol. 18, no. 4, pp. 307-315, 1995.

[13] L. Zhigui and X. Chunhong, "The blow-up rate for a system of heat equations with nonlinear boundary conditions," Nonlinear Analysis: Theory, Methods \& Applications, vol. 34, no. 5, pp. 767778, 1998.

[14] M. Pedersen and Z. Lin, "Blow-up analysis for a system of heat equations coupled through a nonlinear boundary condition," Applied Mathematics Letters, vol. 14, no. 2, pp. 171-176, 2001.

[15] C. Yuan, D. Jiang, D. O'Regan, and R. P. Agarwal, "Multiple positive solutions to systems of nonlinear semipositone fractional differential equations with coupled boundary conditions," Electronic Journal of Qualitative Theory of Differential Equations, no. 13, 17 pages, 2012.

[16] Y. Zou, L. Liu, and Y. Cui, "The existence of solutions for fourpoint coupled boundary value problems of fractional differential equations at resonance," Abstract and Applied Analysis, vol. 2014, Article ID 314083, 8 pages, 2014.

[17] M. Al-Refai and M. Ali Hajji, "Monotone iterative sequences for nonlinear boundary value problems of fractional order," Nonlinear Analysis: Theory, Methods \& Applications, vol. 74, no. 11, pp. 3531-3539, 2011.

[18] T. Jankowski, "Boundary problems for fractional differential equations," Applied Mathematics Letters, vol. 28, pp. 14-19, 2014.

[19] L. Lin, X. Liu, and H. Fang, "Method of upper and lower solutions for fractional differential equations," Electronic Journal of Differential Equations, vol. 2012, 2012.

[20] J. D. Ramirez and A. S. Vatsala, "Monotone iterative technique for fractional differential equations with periodic boundary conditions," Opuscula Mathematica, vol. 29, no. 3, pp. 289-304, 2009.

[21] A. Shi and S. Zhang, "Upper and lower solutions method and a fractional differential equation boundary value problem," Electronic Journal of Qualitative Theory of Differential Equations, vol. 30, 13 pages, 2009.

[22] G. Wang, "Monotone iterative technique for boundary value problems of a nonlinear fractional differential equation with deviating arguments," Journal of Computational and Applied Mathematics, vol. 236, no. 9, pp. 2425-2430, 2012.
[23] Z. Wei, Q. Li, and J. Che, "Initial value problems for fractional differential equations involving Riemann-Liouville sequential fractional derivative," Journal of Mathematical Analysis and Applications, vol. 367, no. 1, pp. 260-272, 2010.

[24] S. Zhang and X. Su, "The existence of a solution for a fractional differential equation with nonlinear boundary conditions considered using upper and lower solutions in reverse order," Computers \& Mathematics with Applications, vol. 62, no. 3, pp. 1269-1274, 2011.

[25] S. Zhang, "Monotone iterative method for initial value problem involving Riemann-Liouville fractional derivatives," Nonlinear Analysis: Theory, Methods \& Applications, vol. 71, no. 5-6, pp. 2087-2093, 2009. 


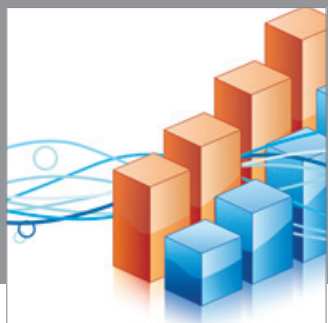

Advances in

Operations Research

mansans

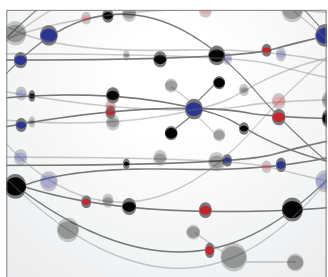

The Scientific World Journal
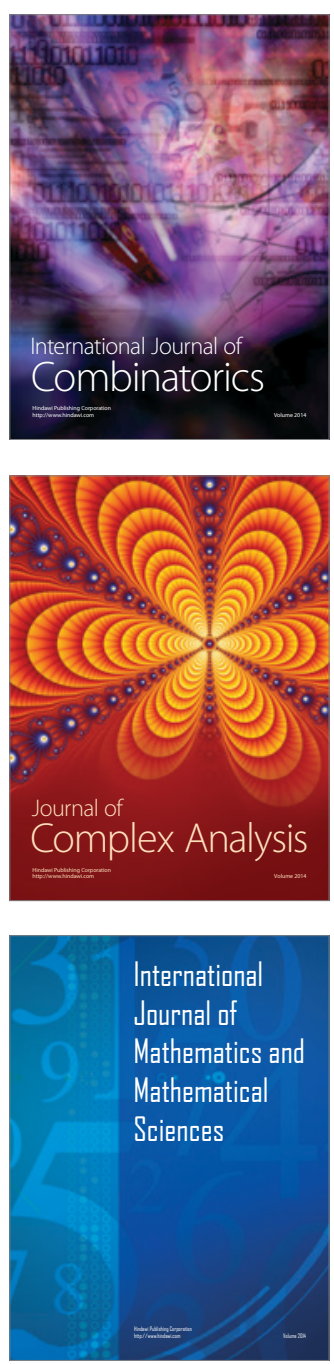
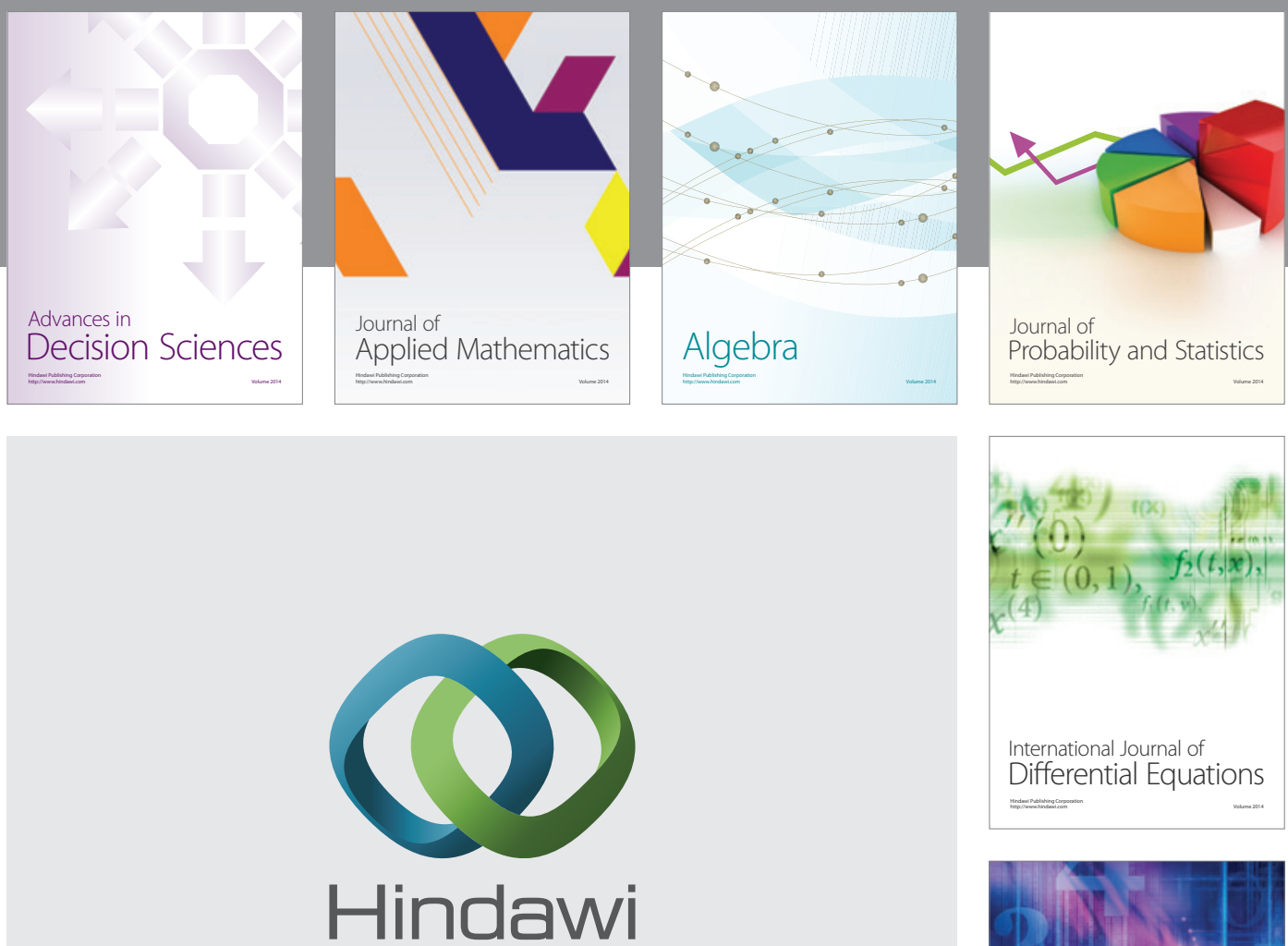

Submit your manuscripts at http://www.hindawi.com
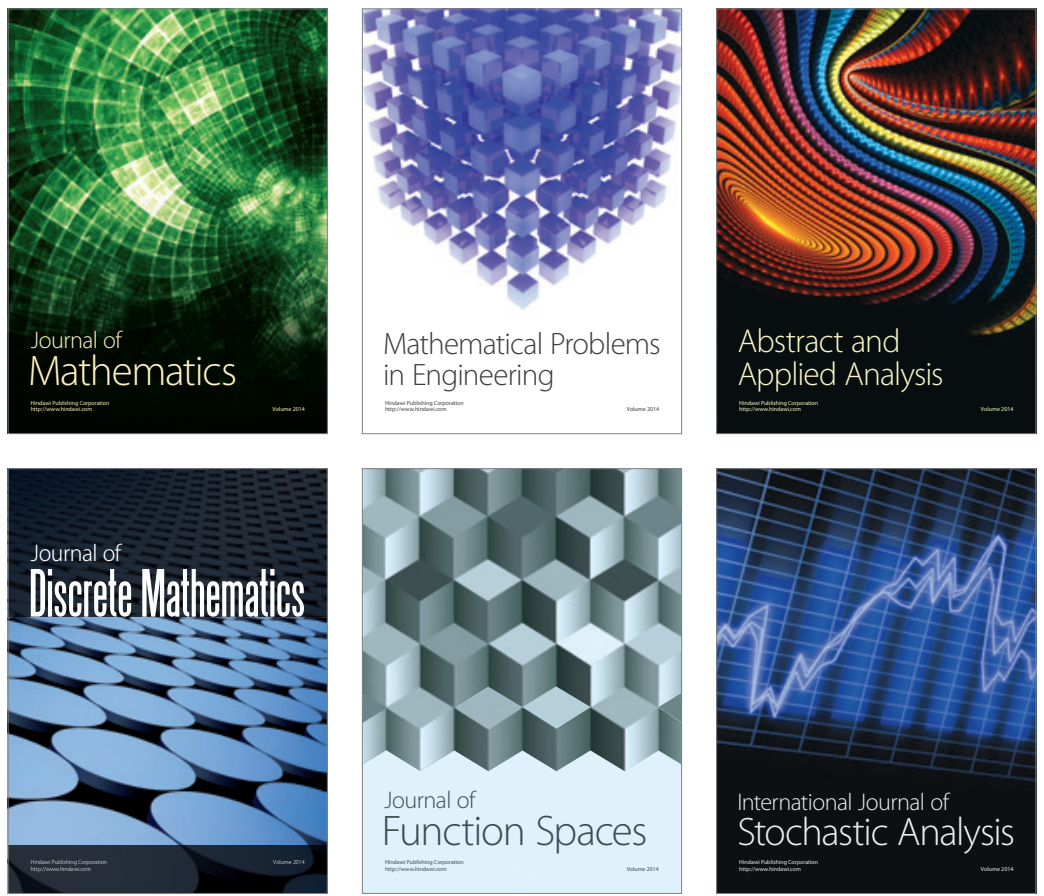

Journal of

Function Spaces

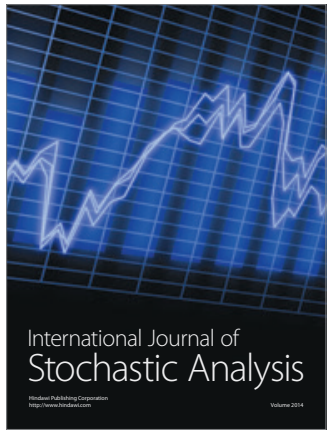

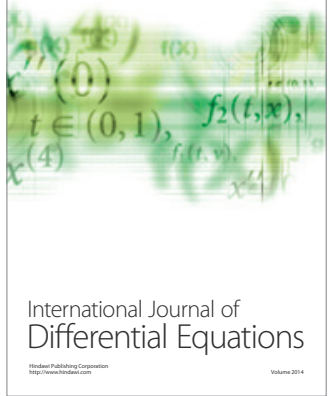
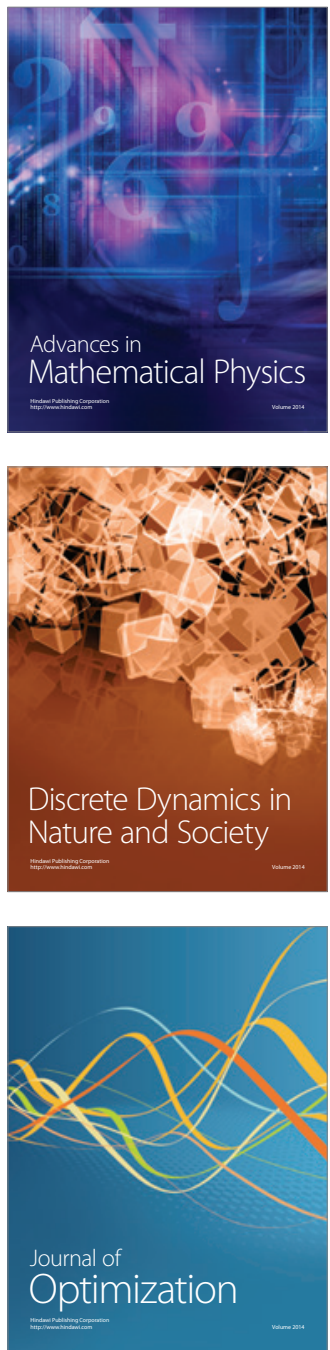